\title{
A UNIQUE LAW FOR IDEAL INCOMPRESSIBLE FLOW WITH PRESERVED PATTERN OF FINITE SEPARATION*
}

BY H. S. TAN (Cornell University)

Prandtl, in treating the rolled up separation surface for a flow around corner (Fig. 1 (a); Ref. 1), and von Kármán, in treating the wake formation for a flow normal to a
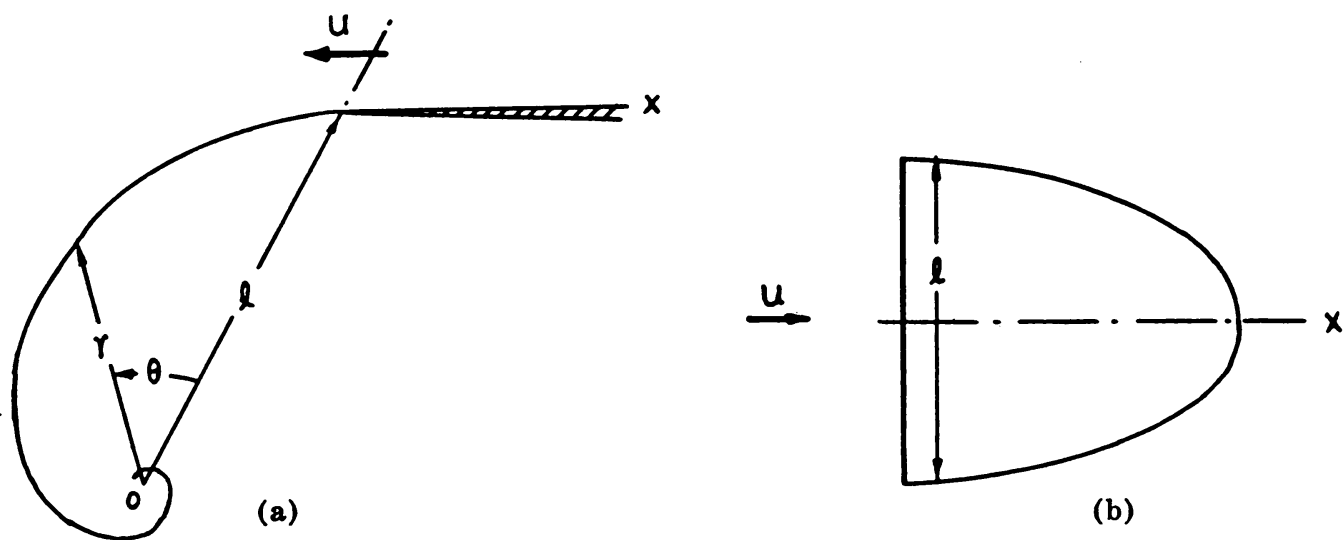

(b)

Fig. 1. (a) Prandtl's case of flow around a corner.

(b) von Kármán's case of flow past a flat plate.

flat plate (Fig. 1(b); Ref. 2), both arrived at a law of fluid motion with stationary flow pattern which can be reduced to the form

$$
U(t)=\frac{U_{0}}{1-\left(a_{0} t / U_{0}\right)},
$$

where we denote by $U(t)$ the velocity at $r=l$ for the first case, and at infinity for the second case, by $a$ the acceleration at the same point; $t$ is the time, and the subscript 0 indicates the initial condition.

It can readily be shown that this law of motion is in fact at once general and unique. The law is general in the sense that it applies to any body shape with well defined separation points, provided there exists a solution. The law is unique because no other fluid motion except this one will produce such a preserved flow pattern.

To prove these statements, let us observe that a satisfactory non-stationary velocity potential $\Phi$ must be of the form

$$
\Phi(x, y, t)=U(t) \phi(x, y)
$$

in order that the flow pattern may not alter with time. This leads directly to the following expression for the pressure:

$$
p=\phi \frac{d U}{d t}+\frac{1}{2} U^{2}\left[\left(\frac{\partial \phi}{\partial x}\right)^{2}+\left(\frac{\partial \phi}{\partial y}\right)^{2}\right] .
$$

${ }^{*}$ Received May 8, 1953. This research was supported by the United States Air Force under Contract No. AF33(038)-21406, monitored by the Office of Scientific Research, Air Research and Development Command. 
Now, $\phi$ is determined by the differential equation

$$
\frac{\partial^{2} \phi}{\partial x^{2}}+\frac{\partial^{2} \phi}{\partial y^{2}}=0
$$

together with the boundary conditions
i) at infinity
ii) on solid boundary
iii) on free boundary

\begin{tabular}{c|c} 
plate flow & corner flow \\
\hline$\phi_{x}=1$ & $\phi_{x}=0$ \\
$\phi_{n}=0$ & $\phi_{n}=0, \phi_{x}(l)=1$ \\
$p=0$ & $\Delta_{n} p=0$
\end{tabular}

The only boundary condition that involves $U$, and hence $t$, is (iii). Since determination of $\phi$ cannot depend on $t$, (iii) must be reducible to a product form, i.e.

$$
L(U) M(\phi)=0 .
$$

But this is evidently the case when and only when $U$ satisfies the following differential equation

$$
\frac{d U}{d t}= \pm k U^{2}, \quad k>0
$$

by virtue of (2).

Integration of (5) leads immediately to the desired law of fluid motion:

$$
U(t)=\frac{U_{0}}{1 \mp k U_{0} t}, \quad U_{0}>0,
$$

where $U_{0}$ is the initial velocity at $r=l$ for Prandtl flow, and at infinity for Kármán flow. Introducing the initial acceleration at corresponding points $a_{0}$, we see that (6) further reduces to

$$
U(t)=\frac{U_{0}}{1-\left(a_{0} t / U_{0}\right)}
$$

which result has been plotted in Fig. 2 .

It may be observed that $a_{0}$ is uniquely determined in terms of $U_{0}$ and a reference length $l$ (where $\phi(l)=1$ in Prandtl flow, and is the plate width in Kármán flow) by certain kinematic considerations. Indeed, for the case of Kármán's plate flow, the condition that the wake must form a closed region leads to the following value for initial acceleration at infinity

$$
a_{0}=0.298 \frac{U_{0}^{2}}{l} .
$$

Whereas for the case of Prandtl's corner flow the condition that the rolled up vortex sheet must coincide with the stream surface leads to the following result

$$
a_{0}=\frac{\exp \left(2 \pi 3^{1 / 2}\right)-1}{2\left[1+\exp \left(-\pi 3^{1 / 2}\right)\right]} \frac{U_{0}^{2}}{l} .
$$


From Fig. 2 it may be noticed that although it is entirely possible to produce a preserved pattern of finite separation both by an accelerated and a decelerated flow

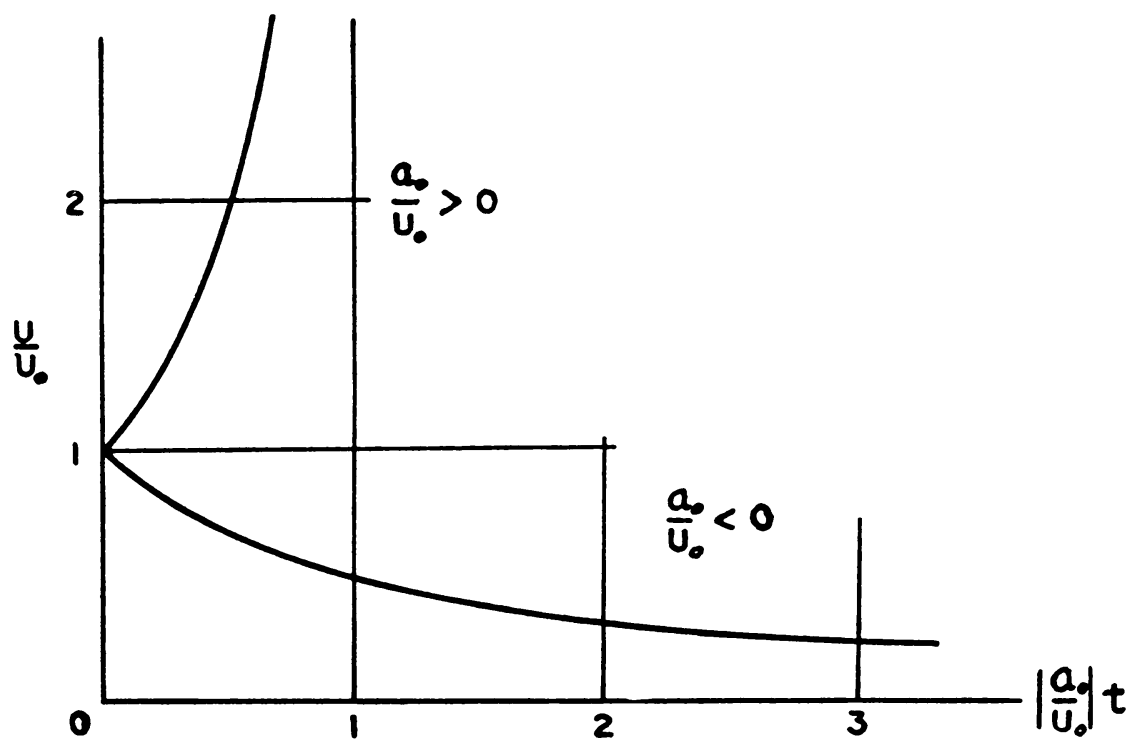

Fig. 2. Variation of flow speed $U$ with time $t$, according to Eq. (7), for accelerated (above) and decelerated (below) flow.

field, yet to maintain such a flow pattern indefinitely, an accelerated field is never adequate; the flow then must be a retarded one.

This analysis definitely rules out the possibility of preserving a finite wake in a stationary flow field, or in any flow field that does not exactly follow the law of motion (7).

\section{REFERENCES}

1. L. Prandtl, Uber die Entstehung von Wirbeln in der idealen Flüssigkeit, mit Anwendung auf die Tragfiugeltheorie and andere Aufgaben, pp. 18-33, Vorträge aus dem Gebiet der Hydro-und Aerodynamik, Innsbruck, 1922.

2. T. von Kármán, Accelerated flow of an incompressible fluid with wake formations, Annali Mat. Pur. Appl. (4) 29, 247-249 (1949).

\section{THE BOUNDARY LAYER ON A QUARTER INFINITE FLAT PLATE*}

\section{By L. TRILLING (Massachusetts Institute of Technology)}

This note discusses the incompressible boundary layer on the surface of a quarter infinite flat plate in the absence of a pressure gradient, generalizing the classical two dimensional Blasius solution [1] and Sears' extension to an arbitrarily yawed plate [2]. It shows that the flow retains free stream direction and Blasius profile at all points of the plate, and that the projection of the constant velocity surfaces on planes parallel

*Received May 21, 1953. 